\title{
Experimental investigations of sedimentation of flocs in suspensions of biological water treatment plants
}

\author{
B. Zajdela ${ }^{1,2}$, A. Hribernik ${ }^{2} \&$ M. Hribersek ${ }^{2}$ \\ ${ }^{1}$ Regional Development Agency Mura Ltd, Slovenia \\ ${ }^{2}$ Faculty of Mechanical engineering, University of Maribor, Slovenia
}

\begin{abstract}
This contribution deals with the movement of flocs in suspensions, as they appear in biological water treatment (BWT) plants. The first part of the work deals with the description of a model BWT plant, followed by the definition of a problem. The greatest attention is given to the geometrical and sedimentation characteristics of solid flocs, key parameters for developing a fast numerical procedure for simulation of flocs' movements. An extensive analysis is presented of floc size distribution and their shapes. Based on the results of experimental investigations, the main geometrical parameters of the flocs are defined and calculated. The second part of the work deals with calculating floc density, based on free settling sedimentation tests and known empirical correlations for drag coefficient.
\end{abstract}

Keywords: biological treatment plants, sedimentation, image analysis, size distribution, drag coefficient, density.

\section{Introduction}

Today, wastewater treatment with the activated sludge is the most widespread process for removing dissoluble substances, small insoluble substances and colloidal organic pollutants from wastewater. The efficiency of the process primarily depends on growing biological biocenoze, consuming and consequently removing unwanted substances, and secondarily on accumulating the activated sludge into sludge flocs. The sludge flocs are subjected to circulation inside the wastewater processing tank and in the final phase of the flocs' sedimentation process, separating the cleaned water from the accumulated 
impurities in the sludge [1]. The rate of sedimentation depends on the properties of the flocs, such as size, shape, density, permeability, number density and type of wastewater (industrial, municipal). In the presented paper, the greatest attention is therefore given to determining the size distribution and main geometrical parameters of the sludge flocs, by means of image analysis. Additionally, free settling tests in connection with empirical models for determining of the drag coefficient $\left(C_{D}\right)$ are used, in order to evaluate the densities of the flocs.

\section{Materials and methods}

\subsection{Biological treatment plant}

The performed analysis of the sludge flocs concentrated on the data, obtained from the wastewater samples of the Water treatment plant in Lendava, Slovenia. The wastewater samples, taken from the plant, were analysed within a time period of 1-3 days. The samples were diluted and kept at $20^{\circ} \mathrm{C}$. The analysed wastewater was a mixture of technological and municipal waste water in the ratio of 75:25. Several analyse of various waste water samples were needed in order to obtain accurate and representative results.

\subsection{Image analysis system}

Wastewater samples were investigated using a Nikon stereoscopic microscope, which allowed us to see the samples at 20-126x magnification. Pictures were taken with a high-resolution Sony CCD videocamera, computer controlled using the Lucia M (Nikon, Japan) software package. Lucia $M$ is a colour-image analysis software system, handling and analyzing High Color (3x5 bits for RGB components) digital images of typical $752 \times 524$ pixels resolution. Lucia $M$ basically recognizes two types of images - binary and colour. Binary images have two possible values, 0 for background and a maximum of 62 for objects and structures. Binary images are produced by segmentation functions such as threshold and are often referred to as segmented images.
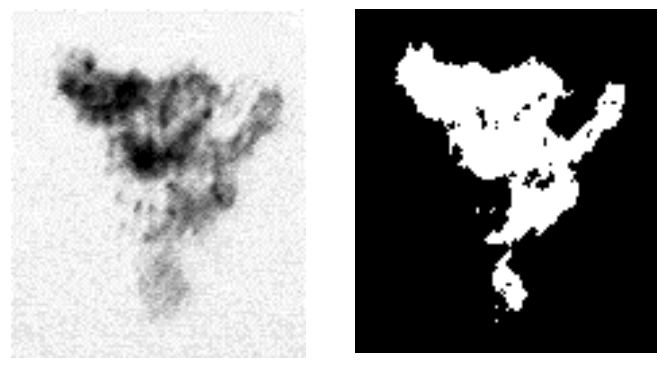

Figure 1: Colour and binary images of sludge floc. 
Once the threshold value is defined the object can be clearly distinguished from the background, such segmented images (fig. 1) being used for automated shape and size measurements (area, diameter, and perimeter).

\subsection{Preparation of samples}

The sludge sample must be sufficiently diluted to avoid saturation of the image and the liquid layer thickness must be small enough to enable the use of relatively high magnifications $(80 x)$, focusing on a thin plane. By experimenting with different levels of dilution we discovered that some areas overlap even at the dilution ratio 1:15. Further diluting increased the isolation of the flocs and at a dilution of 1:20 there was no floc overlap. As quoted in Grijspeerdt and Verstraete [2], it is inherent to image analysis that smaller objects produce larger measurement errors. Objects are approximated by square pixels, resulting in a less accurate measurement of smaller objects. This problem could be solved by imposing a lower limit on the number of pixels in an object. Russ noted that $0.1 \%$ of objects comprising a number of pixels smaller than $0.1 \%$ of the total number of pixels of an image should not be taken into account [3]. In our case the size of the picture was $752 \times 524$ pixels and so $0.1 \%$ of the picture represented 394 pixels. In the case of $80 \mathrm{x}$ magnification this means that $0.1 \%$ of the picture was within an area of $0.001646 \mathrm{~mm}^{2}$, therefore, flocs smaller that this area were not analysed.

\subsection{Drag on non-spherical particles}

In general, the force balance for a floc moving steadily in an infinite medium can be described as follows [4]:

$$
\frac{\rho_{f}-\rho_{w}}{\rho_{p}-\rho_{w}}=1-e=\frac{3 \rho_{w} \Omega C_{D}}{4 g\left(\rho_{p}-\rho_{w}\right) d_{f}} v^{2}
$$

$\rho_{\mathrm{f}}$ is floc density, $\rho_{\mathrm{w}}$ is the density of the water and $\rho_{\mathrm{P}}$ is the density of the primary particles comprising the floc, $C_{D}$ is the drag coefficient, $\mathrm{g}$ is gravitational acceleration, $v$ is the terminal velocity, $d_{f}$ is the diameter of the floc, and $\mathrm{e}$ is porosity of the floc. In most studies, $\rho_{\mathrm{P}}$ is assumed to be equal to $\rho_{\mathrm{S}}$, the dried solid density $[5,6]$. $\Omega$ is the ratio of the resistance experienced by a porous floc to that of an equivalent solid sphere. Floc porosity is neglected in many studies [5, 7] and $\Omega$ is simply set to 1 . The $\rho_{\mathrm{f}}$ is difficult to measure experimentally, therefore we decided to compute it based on data (velocity) obtained from the sedimentation properties of the flocs, as follows:

$$
\rho_{f}=\frac{\left(3 \cdot \rho_{w} \cdot 1 \cdot C_{D} \cdot v^{2}\right)}{\left(4 \cdot g \cdot d_{f}\right)}+\rho_{w}
$$

In order to evaluate the right hand side in eqn. (2) geometrical data and data on the $C_{D}$ of the flocs must be known. In the present work, the $C_{D}$ correlations 
were used of different authors, who have performed comparison studies. The diameters and volumes of the flocs were calculated based on image analysis. Sedimentation tests were performed for terminal velocity.

In general, $C_{D}$ is a function of Reynolds number (Re) and floc sphericity. An equal volume sphere diameter is needed as the characteristic linear dimension in order to evaluate the $C_{D}$. As a consequence, the sphericity $(\psi)$ is commonly introduced in order to quantify the extent of the particle's deviation from the spherical-shaped (Wadell [8]):

$$
\psi=\frac{6}{d_{s}}\left(\frac{V_{p}}{A_{p}}\right)=\left(6^{2} \pi\right)^{\frac{1}{3}} \frac{V_{p}^{\frac{2}{3}}}{A_{p}}=\frac{4.84 V_{p}^{\frac{2}{3}}}{A_{p}}
$$

where $V_{p}$ is volume of the floc and $A_{p}$ is the surface area of the floc. Corey's shape factor $(\beta)$ can be used as an alternative to sphericity, with $a>b>c$ the lengths of the three principal axes of the floc:

$$
\beta=c /(a b)^{1 / 2}
$$

When dealing with the sedimentation of particles in an infinite medium, the particle Reynolds number is defined as:

$$
\operatorname{Re}=\frac{\psi v \rho_{w} d_{f}}{\eta}
$$

where $v$ is the terminal velocity of the floc in the medium of dynamic viscosity $\eta$ which, due to the incorporation of sphericity, differs from the classical equation for spheres:

$$
\operatorname{Re}=\frac{v \rho_{w} d_{f}}{\eta}
$$

The starting point for derivation of a suitable expression for the $C_{D}$ of a nonspherical particle is the well-known expression for the sphere in a laminar flow:

$$
C_{D}=\frac{24}{\operatorname{Re}}
$$

In the case of non-spherical flocs it is most appealing to derive a correction to the expression (7). Haider and Levenspiel [9] developed an expression for $C_{D}$ for nonporous spherical and non-spherical flocs in incompressible media:

$$
C_{D}=\frac{24}{\operatorname{Re}}\left(1+A \operatorname{Re}^{B}\right)+\frac{C}{1+\frac{D}{\operatorname{Re}}}
$$


with the values of $\mathrm{A}, \mathrm{B}, \mathrm{C}$ and $\mathrm{D}$ as model constants and the equal volume sphere diameter used in the definition of Re. This method is applicable for $\mathrm{Re}<$ $2.6 \times 10^{5}$, uncertainty is predicted to be $15-20 \%$. They also proposed the following equation for flocs of $\Psi>0.67$ :

$$
\begin{aligned}
& C_{D}=\frac{24}{\operatorname{Re}}\left[1+\left\{8.1716 \cdot e^{(-4.0655 \psi)}\right\} * \operatorname{Re}^{(0.0964+0.5565 \psi)}\right] \\
& +\frac{73.69 \cdot e^{(-5.0748 \psi)}}{\operatorname{Re}+5.378 \cdot e^{(6.2122 \psi)}}
\end{aligned}
$$

Ganser [10] assumed that every floc experiences a Stokes's regime where drag is linear in velocity and a Newton's regime where drag is proportional to the square of velocity. He thus introduced two shape factors $K_{1}$ and $K_{2}$ applicable in both the Stokes and Newton regimes, respectively, in the following drag correlation:

$$
\frac{C_{D}}{K_{2}}=\frac{24}{\operatorname{Re} K_{1} K_{2}}\left\{1+0.1118\left(\operatorname{Re} K_{1} K_{2}\right)^{0.6567}\right\}+\frac{0.4305}{1+\frac{3305}{\operatorname{Re} K_{1} K_{2}}}
$$

with $\mathrm{Re}$ based on the equal volume sphere diameter, and $\mathrm{K}_{1}$ and $\mathrm{K}_{2}$ as unique functions of sphericity:

$$
\begin{gathered}
K_{1}=\left[\left(d_{n} / 3 d_{f}\right)+(2 / 3) \psi^{-0.5}\right]^{-1} \\
K_{2}=10^{1.8148(-\log \psi)^{0.5733}}
\end{gathered}
$$

where $d_{n}$ is the equally projected area of the circles diameter.

Swamee and Ojha [11] employed the equal volume sphere diameter and used the so called Corey shape factor $(\beta)$ in the following drag expression:

$$
C_{D}=\left[\frac{48.5}{\left(1+4.5 \beta^{0.35}\right)^{0.8} \operatorname{Re}^{0.64}}+\left\{\left(\frac{\mathrm{Re}}{\operatorname{Re}+100+100 \beta}\right)^{0.32} \frac{1}{\beta^{18}+1.05 \beta^{0.8}}\right\}\right]
$$

which was stated to be applicable within the range $0.3<\beta<1$ and $1<\operatorname{Re}$ $<10.000$.

Finally, Chien [12] proposed the following simple expression for drag, originating from petroleum engineering literature:

$$
C_{D}=(30 / \mathrm{Re})+67.289 \cdot e^{(-5.03 \psi)}
$$

for $0.2 \leq \psi \leq 1$ and $\operatorname{Re}<\sim 5000$, where the equal volume sphere diameter was used. 


\subsection{Floc size distribution}

The presented image analysis system was applied for floc size distribution measurements.

Automatic processing was performed by allowing an appropriate threshold for the transformation of pictures into binary images. The results obtained were morphological characteristics of the flocs. From the standard deviation $\sigma$ analysis the minimum number of flocs in a representative sample was determined to be $\mathrm{n}>400$. Approximately 18 photos were needed to reach the minimum size for a representative sample. The basic sample consisted of 729 flocs with the flocs surface areas within the range from $0.0000042 \mathrm{~mm}^{2}$ to $0.0759 \mathrm{~mm}^{2}$. According to the " $<0,1 \%$ " rule, we excluded all those flocs whose areas were smaller than $0.001646 \mathrm{~mm}^{2} .72$ flocs remained and were separated into 7 size classes. The details are presented in fig. 2, showing flocs' frequency distribution and cumulative flocs' surface area distribution. Following from our observations, the average floc's equivalent diameter is $0.086837 \mathrm{~mm}$ and the average floc's surface area is $0.007845 \mathrm{~mm}^{2}$.

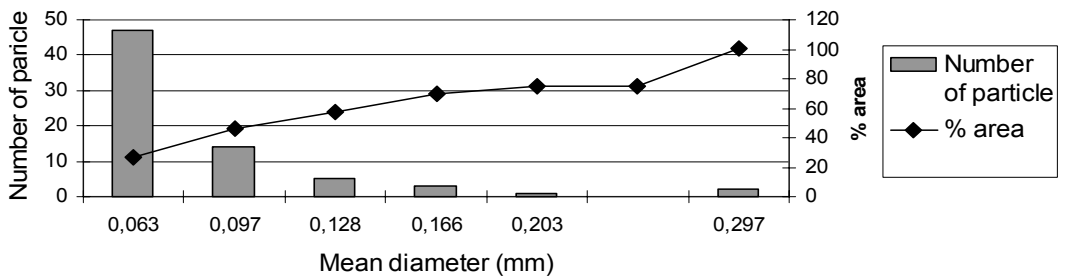

Figure 2: Flocs frequency distribution and cumulative flocs surface area distribution.

\subsection{Main geometrical parameters}

Only observation of floc's plane projection is possible sing floc image analysis. No information on a floc's volume is necessary for the calculation of floc sphericity, $C_{D}$ and density. Due to the different shapes of flocs it is very difficult to determine a representative three dimensional floc shape and volume. A large number of flocs were, therefore, analysed. Each individual floc was rotated in order obtain floc images in three orthogonal planes (fig. 3) and then an attempt was made to create a 3D shape of a floc using its 3 orthogonal 2D images.

The 3D floc's shape was approximated by an equivalent cuboid whose projections on the orthogonal planes were equal to those of the floc. The transformation can be written using a simple model as shown in fig. 3 .

14 randomly selected flocs from the diluted sample $(1: 20)$ of waste water were processed in this way. Three replications were made for each individual floc and the proportions $A=P 1 \cdot A, \quad B=P 2 \cdot A, \quad C=P 3 \cdot A$ with $P 1, P 2$ and $P 3$ as weighting factors, were estimated. Weighting factors $P 1$ and $P 2$ deviated only slightly from their average values, while the scatter of weighting factor $P 3$ 
was much bigger. In spite of this, the average values of weighting factors were used for the sake of simplicity and the ratio of the equivalent cuboid surfaces was found to be $A: B: C=P 1: P 2: P 3=1: 0.89: 0.69$.

Experiments showed that the flocs always set themselves up in a way that the projection surface in the horizontal plane which corresponds to the projection $A$ (fig. 3) was the largest. Therefore, it is possible to estimate the sizes of projection planes $B$ and $C$ by measuring the projection of flocs in plane $A$ and considering the ratio $A: B: C$. The edges of the equivalent cuboids $a, b$ and $c$ are then determined and the volume of each floc is estimated as $V=a \cdot b \cdot c$. Finally the shape factors $\psi$ and $\beta$, necessary in empirical equations for $C_{D}$, are calculated. Since the constant ratio $A: B: C$ was applied, the shape factors were equal for all flocs.

Eqn. (3) was applied for the prediction of floc sphericity $\psi$, which was found to be $\psi=0.802$ for the sampled wastewater. Corey factor $\beta$ of flocs was calculated from eqn. (4) as $\beta=0.86$.

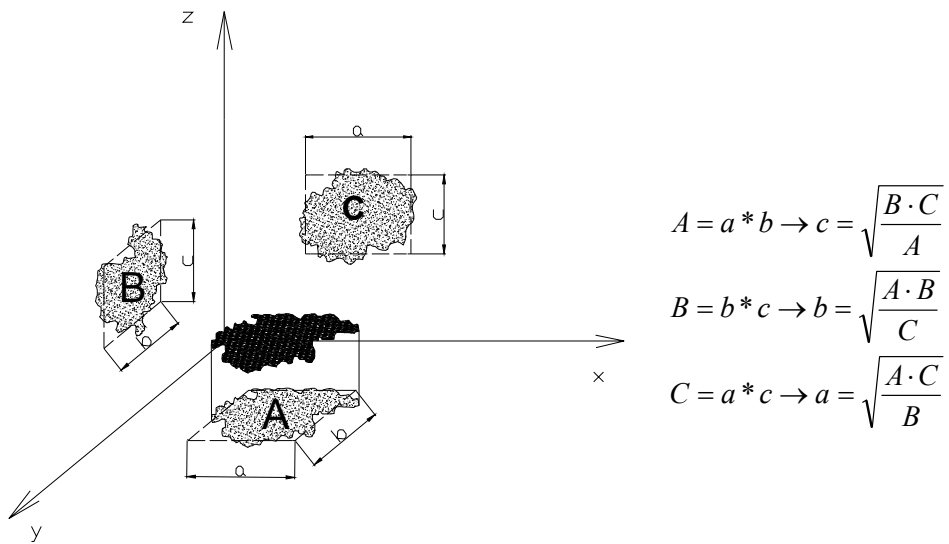

Figure 3: $\quad$ Equivalent cuboid; $A, B, C$ - projection areas.

\subsection{Drag coefficient $\left(C_{D}\right)$}

A large number of free settling tests were performed and terminal settling velocities of flocs were measured in order to estimate the suitability of the presented $C_{D}$ models given by eqns. (7) - (14)

A $50 \mathrm{ml}$ glass cylinder was used and the time of travel of a single floc on a chosen distance was measured. Three replications of each settling test were made and the average terminal settling velocities was calculated. Only larger flocs were selected for tests in order to improve visualisation quality. The results are shown in fig. 4. The terminal settling velocity of the flocs varied between 2.5 and $4.7 \mathrm{~mm} / \mathrm{s}$. It increases with the flocs diameter (size), which agrees with the results of other authors [13]. 


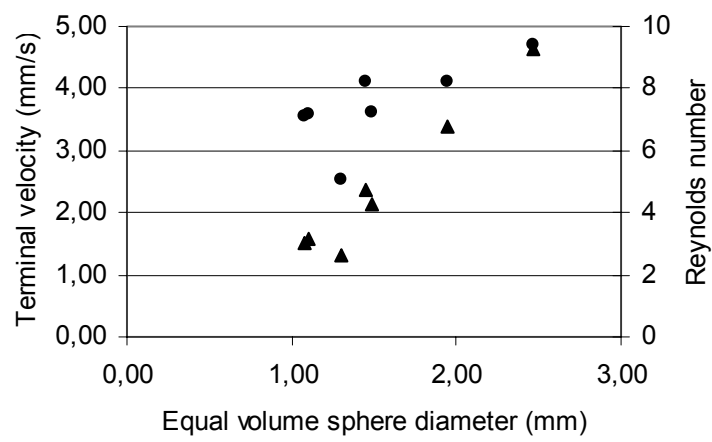

- Equal volume sphere diameter vs terminal velocity

$\Delta$ Equal volume sphere diameter vs Reynolds number

Figure 4: Terminal velocity and Reynolds number of flocs.

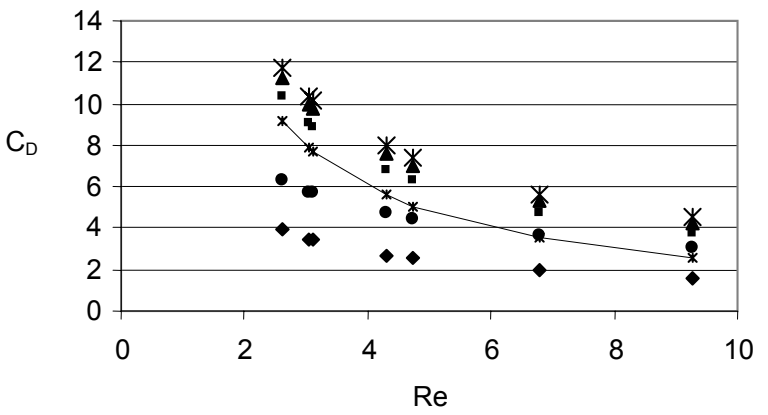

- $C_{D}$ Ganser

- $C_{D}$ Chien

\ $\mathrm{C}_{\mathrm{D}}$ Haider1

$* \mathrm{C}_{\mathrm{D}}$ Haider2

$\rightarrow C_{D}$ laminar

- $C_{D}$ Swamee in Ojha

Figure 5: $\quad$ Comparison between different $C_{D}$ models.

Data obtained from the free settling tests were used to predict the $C_{D}$, fig. 5 . The differences between individual models were as high as $300 \%$. Models proposed by Chien (eqn. (14)) and Haider (eqns. (8) and (9)) result in higher $C_{D}$ values than the perfect sphere model (eqn. (7)), while the models by Ganser (eqns. (10)-(12)) and Swamee (eqn. (13)) predict lower $C_{D}$ values.

The $C_{D}$ decreases with $\mathrm{Re}$ and the differences between the individual models become smaller. However, it should not be neglected that only the largest flocs were studied by free settling tests. An average floc and its Re are much smaller, thus its $C_{D}$ is substantially higher than those presented in fig. 5, and an appropriate $C_{D}$ model is essential. It is evident, that models proposed by Ganser and Swamee under-predict $C_{D}$ value (it should not be under the $C_{D}$ value of the sphere). Models by Haider and Chien predict more reliable $C_{D}$ values which are very close together $( \pm 10 \%)$. Because of its simplicity and a wide application range the Chian model (eqn. (14)) was chosen for application in our model.

\subsection{A floc's density}

A floc's density plays an important role in the sedimentation process. The sedimentation of suspended matter in the water is possible only if its density is higher than the water density. Otherwise it floats. 
Using eqn. (2) the flocs density can be predicted if the floc's terminal velocity, $C_{D}$ and equivalent diameter are known. The densities of the flocs used in the free settling tests were estimated in this way. The measured terminal velocities and the floc's equivalent diameter were applied and $C_{D}$ predicted by the Chien model was used. The results are presented in fig. 6 , where the difference in the floc's and water densities is plotted against the floc's diameter. As can be seen the floc's density decreases with the floc's size. Flocs are formed from a large number of primary flocs with the in-between free space filled by water. Therefore, the floc density decreases with its size, which was also proven by other authors [13].

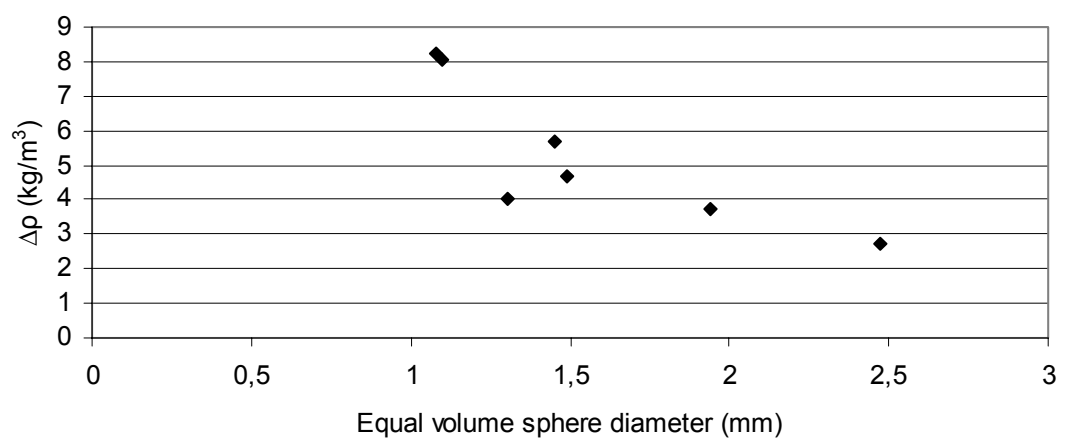

Figure 6: Difference of flocs and water density against flocs diameter.

\section{Conclusion}

The properties of the activated sludge flocs were examined. An image-analysis system was applied to determine floc size distribution. A representative waste water sample comprising 729 flocs was acquired. Those flocs larger than $0.1 \%$ of the whole image area were selected from this sample and analyzed. The 3D floc shape was approximated by an equivalent cuboid whose projections on the orthogonal planes were equal to those of the floc. The ratio of the equivalent cuboid surfaces was determined and used to predict floc volume and shape factors by measuring only the horizontal projection areas of the flocs. Mean floc shape factors were found to be $\psi=0.802$ and $\beta=0.86$.

A large number of free settling tests were performed and the obtained results were applied in six different $C_{D}$ models. The model proposed by Chien was selected as the most appropriate and applied in the floc-free settling model for floc density computation. A floc's density was found to vary with its size. The density of the smallest analysed floc was $1 \%$ higher and the density of the largest analysed floc was only $0.3 \%$ higher than density of the water.

The obtained results present a starting point for numerical simulation of a floc's motion in water, where the density of the floc, its size and its drag coefficient are key parameters for particle path computations. 


\section{References}

[1] Roš, M., Biološko čiščenje odpadne vode, GV založba, (2001), (in slovene).

[2] Grijspeerdt, K. \& Verstraete, W., Image analysis to estimate the settleability and concentration of activated sludge, Wat. Res. 31, pp. 1126-1134, 1997.

[3] Russ, J. C., Computer - Assisted Microscopy: the Measurement and Analysis of Images, Plenum Press, New York, 1990.

[4] Huang, H., Porosity - size relationship of drilling mud flocs: fractal structure, Clay Clay Miner 41, pp. 373 - 379, 1993.

[5] Tambo, N. \& Watanabe, Y., Physical characteristics of flocs. 1. The floc density function and aluminum floc, Wat. Res. 13, pp. 409 - 419, 1979.

[6] Li, D.-H. \& Ganczarczyk, J. J., Stroboscopic determination of settling velocity, size and porosity of activated sludge flocs, Wat. Res. 21, pp. 257 $-262,1987$.

[7] Concha, F. \& Almerdra, E. R., Settling velocities of particulate system, 1. Settling velocities of individual spherical particles, Int. J. Min. Process. 5, pp. $349-367,1979$.

[8] Wadell, H., Sphericity and roundness of rock particles, J. Geol 41, pp. $310-331,1933$.

[9] Haider, A. M., Levenspiel, O., Drag coefficient and terminal velocity of spherical and nonspherical particles, Power Technol. 58, pp.63 - 70, 1989.

[10] Ganser, G. H., A rational approach to drag prediction of spherical and nonspherical particles, Power Technol. 77, pp. 143 - 152, 1993.

[11] Swamee, P. K. \& Ojha, C. P., Drag coefficient and fall velocity of nonspherical particles, J. Hydraul. Eng. 117, pp. 660 - 667, 1991.

[12] Chien, S. F., Settling velocity of irregularly shaped particles, SPE Drilling and Completion, pp. $281-289,1994$.

[13] Lee, D. J., Chen, G. W. \& Hsieh, C. C., On the fre-settling test for estimating activated sludge floc density, Wat. Res. 30, pp. 541 - 550, 1996. 